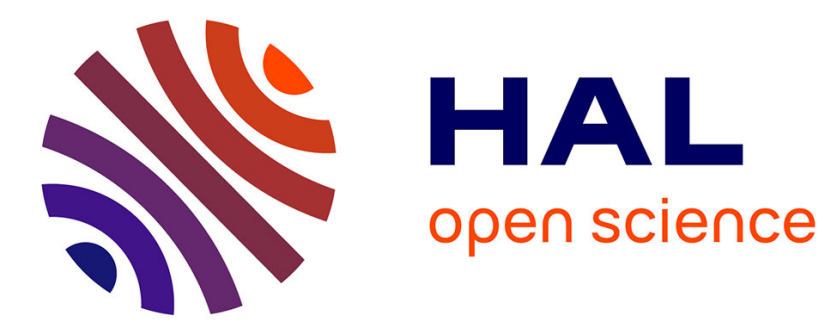

\title{
Délimiter les banlieues de Beyrouth. Des noms et des périmètres \\ Éric Verdeil
}

\section{To cite this version:}

Éric Verdeil. Délimiter les banlieues de Beyrouth. Des noms et des périmètres. Des banlieues à la ville, espaces et acteurs de la négociation urbaine, Oct 2011, Beyrouth, France. n.p. halshs-00644859v2

\section{HAL Id: halshs-00644859 \\ https://shs.hal.science/halshs-00644859v2}

Submitted on 3 Sep 2013

HAL is a multi-disciplinary open access archive for the deposit and dissemination of scientific research documents, whether they are published or not. The documents may come from teaching and research institutions in France or abroad, or from public or private research centers.
L'archive ouverte pluridisciplinaire HAL, est destinée au dépôt et à la diffusion de documents scientifiques de niveau recherche, publiés ou non, émanant des établissements d'enseignement et de recherche français ou étrangers, des laboratoires publics ou privés. 


\section{Presses de I'Ifpo}

Des banlieues à la ville | Élisabeth Longuenesse, Caecilia Pieri

\section{Délimiter les banlieues de Beyrouth. Des noms et des périmètres}

Delimiting Beirut's Suburbs. Names, perimeters, numbers

ترسيم حدود ضواحي مدينة بيروت . أسماء وحدود

\section{Éric Verdeil}

\section{Résumé}

Cette communication propose l'analyse de quelques découpages et délimitations des banlieues de Beyrouth et de leurs dénominations, avec pour objectif de mettre au jour leurs enjeux cognitifs et politiques. Dans le cas libanais, elle renvoie à l'existence d'un conflit entre l'État central, principal détenteur des compétences urbanistiques, et différents acteurs et pouvoirs locaux, en particulier les municipalités.

This paper analyses some apportionments and delimitations of Beirut's suburbs as well as their denominations, with the aim to update their political and cognitive stakes. In the case of Lebanon, it refers to an existing conflict between the central State, which is the main holder of urban competencies, and different local actors and powers, especially municipalities.

تقترح هذه الورقة تحليل بعض تقطيعات وحدود ضو احي بيروت وتسمياتها، وذلك بهدف تحديث تحدياتها الإدر اكية والسياسية. في الحالة اللبنانية، تحيل هذه

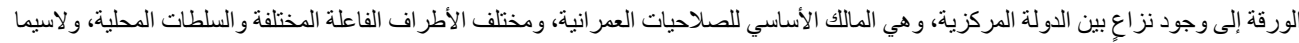

\section{Entrées d'index}

Mots clés :

délimitation, banlieue, municipalités

Keywords :

delimitations, suburbs, municipalities, Beirut

Lieux :

Beyrouth

: تهرس الكلمات المفتاحية:

ضو احي, وحدود, بيروت, بلديات 


\section{Texte intégral}

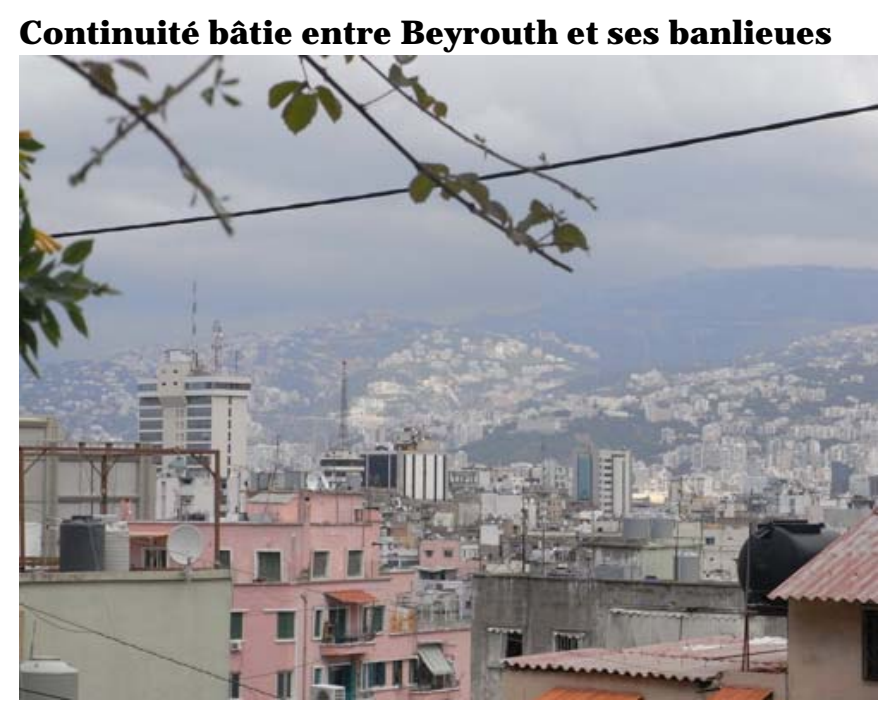

Photo C. Pieri 2011

Le programme « Les mots de la ville » du CNRS a consacré plusieurs de ses opus aux questions de dénomination, au prisme des relations entre le centre et la périphérie ; en particulier, il a examiné comment la périphérie en construction et comme territoire habité constitue un lieu d'élaboration de nouvelles dénominations pour des lieux ou de nouveaux espaces urbains (RIVIĖRE D'ARC 2001). La question du nom des nouvelles entités urbaines nées de la croissance et du débordement des villes mères a constitué un débat central pour les géographes au cours du Xxe siècle, à travers les usages controversés et non stabilisés du mot métropole et les inventions de conurbation, mégapole, mégalopole, etc. (DAGORN 2003 ; LAMARRE et MAUMI 2010 ; REEDER 2010). Toutefois, la dénomination individuelle des grandes villes semble avoir été moins systématiquement explorée. L'accolement de l'adjectif « grand » au nom de la ville mère, qu'on retrouve en anglais sous une forme plus complexe (Great ou Greater) s'est très largement imposé dans les usages urbains et notamment dans la gestion urbaine depuis le début du xxe siècle (MEURIOT 1919). Dans un autre domaine, c'est un autre usage qui domine : les statisticiens de la Banque mondiale ou les géographes comme François-Moriconi Ebard (MORICONI-EBRARD et HUBERT 2000 ; MORICONI-EBRARD 1993), œuvrant au dénombrement de grandes agglomérations, adoptent une pratique plus simple : ils placent, sous le nom de la ville centre, l'ensemble de la population des unités qui la composent (une exception étant peut-être constituée par de très grandes conurbations comme Tokyo-Yokohama où l'on garde parfois les deux noms).

Derrière ces dénominations émergent plusieurs enjeux, et en particulier la question de la délimitation spatiale des espaces urbains, donc la définition de critères d'agrégation ou d'exclusion, ainsi que la question de la nature des liens établis entre les lieux placés sous le même nom. S'agit-il de l'établissement de nouvelles relations d'ordre politique, et sur quelle base le pouvoir est-il alors réparti ? Cela se traduit-il par l'avènement de nouveaux statuts administratifs, par la création de nouvelles instances de type pluricommunal, comme on les appelle en France ? S'agit-il uniquement d'une vision théorique de l'espace urbain comme formant une unité de fonctionnement, mais sans traduction politique ni pratique ? On connaît pourtant les effets performatifs très souvent induits à partir de désignations présentées comme uniquement descriptives (DEPAULE 2006).

3 Cet article revient sur un dossier qui n'est pas tout à fait inédit pour les chercheurs qui se sont intéressés à Beyrouth : la constitution d'une agglomération capitale qui excède largement le périmètre de la ville-centre, et celle des formes de gouvernance de cet espace, notamment du point de vue urbanistique et des services urbains. Il existe une longue tradition de travaux scientifiques consacrés à cette question, notamment chez les géographes (BOURGEY 1970 ; ARNAUD 1997 ; DAVIE 1993), en parallèle et en écho avec les études et les propositions des urbanistes ayant travaillé à l'aménagement de Beyrouth (d'Écochard à l'IAURIF - Institut d'aménagement et d'urbanisme de la région Île-deFrance). Dans ces travaux et réflexions, délimitation spatiale et mesure démographique 
(et économique) s'articulent étroitement au projet d'aménagement, visant notamment le contrôle de l'extension urbaine. Il nous parait intéressant de revisiter ce corpus aujourd'hui ; en effet, la question d'une région métropolitaine à gérer, si angoissante pour les urbanistes (libanais et étrangers), des années 1960 aux années de la reconstruction, semble avoir perdu de son actualité. Du reste, l'administration des statistiques (Central Administration of Statistics - CAS), qui avait donné la seule véritable définition institutionnelle à cette abstraction qu'était la région de Beyrouth, n’a pas publié de données correspondant à ce périmètre urbain dans sa dernière enquête-ménages (2008). Est-ce le signe que la pratique de la gestion urbaine et de l'urbanisme s'est transformée, laissant émerger d'autres acteurs et d'autres priorités ? C'est sur cette hypothèse exploratoire que se construit cette communication : délimitation et dénomination de l'agglomération de Beyrouth dans son ensemble ne semblent plus guère un enjeu politique ; on peut l'analyser de pair avec la montée en puissance des municipalités, notamment des banlieues, dans la gestion urbaine. Dès lors, la question de l'échelle pertinente de l'aménagement semble se déplacer vers de nouveaux enjeux de dénomination et de délimitation territoriale ; on constate notamment une émergence de nouvelles formes de coopération supramunicipales, une reconfiguration des territoires de la gestion urbaine (services urbains) comme l'eau, les déchets ou l'électricité, et tout cela compose de nouvelles géométries qui s'articulent à celle de l'échelon municipal. En somme, cette lecture en termes de périmètres et de noms permet de révéler de nouveaux jeux d'acteurs dans l'urbanisme libanais.

\section{Délimiter Beyrouth, un projet d'urbanistes sans traduction politique ; retour dans I'histoire}

4 L'objectif de cette première partie est de revenir sur quelques avatars de la question de la dénomination et de la construction du territoire beyrouthin des années 1950 aux années 1980. Cette période correspond en effet d'abord à la construction de l'urbanisme national libanais, placé au service de la construction d'un État dans les années de l'indépendance, et dont le chéhabisme fut le moment pivot, puis à un long moment de remise en question (VERDEIL 2010). Dans ces deux moments, on peut identifier des tensions autour du découpage et de la dénomination de l'agglomération formée par Beyrouth et ses banlieues. Les compromis trouvés renvoient aux rapports de force et aux négociations entre les parties prenantes : l'État et ses administrations du Plan et de l'urbanisme et les municipalités de Beyrouth et de ses banlieues dans la première période ; l'État et l'administration de l'urbanisme et les pouvoirs miliciens durant la guerre.

5 En premier lieu, il est nécessaire de rappeler l'ancienneté de la délimitation entre Beyrouth et ses environs à l'époque ottomane (fig. 1). En effet, le territoire de la wilaya de Beyrouth a été déterminé en 1888 (DAVIE 1993). Il présentait l'originalité d'être un territoire discontinu, la mutasarrifiya ayant en effet un statut autonome et ne relevant donc pas de l'autorité de Beyrouth. Cette division très forte est restée durablement inscrite dans la réalité administrative libanaise : la capitale forme une mohafaza, et le Mont-Liban, qui inclut les banlieues de Beyrouth, en forme une autre, dirigée chacune par un mohafez distinct (fig. 2). Nombre de découpages administratifs se calquent sur cette division et font sentir leurs effets dans la vie quotidienne, dont l'un des plus sensibles est la différence d'alimentation en électricité entre la capitale stricto sensu et les banlieues, sévèrement rationnées comme le reste du pays.

Fig. 1- Wilaya de Beyrouth et mutasarrifiya du Mont-Liban 


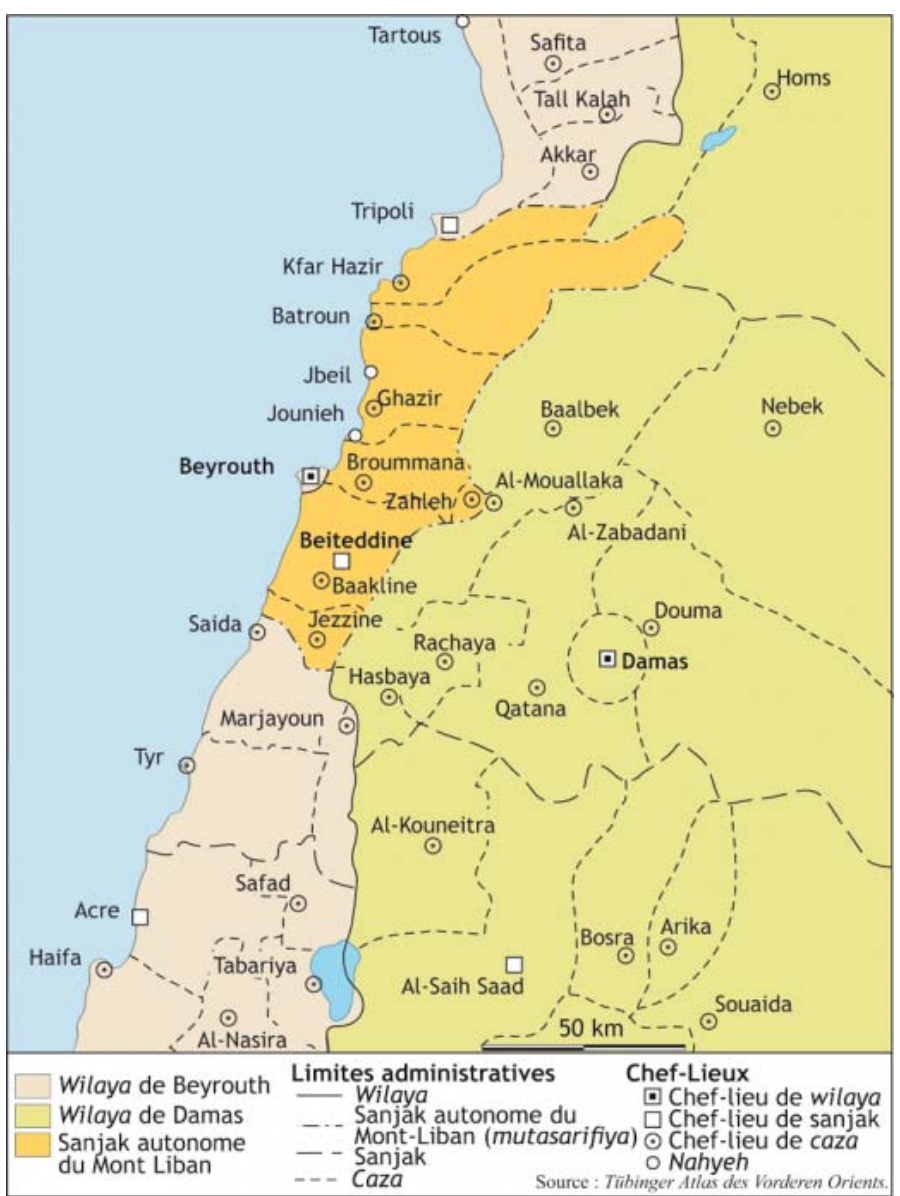

Fig. 2 - L'organisation administrative du territoire libanais au xxe siècle
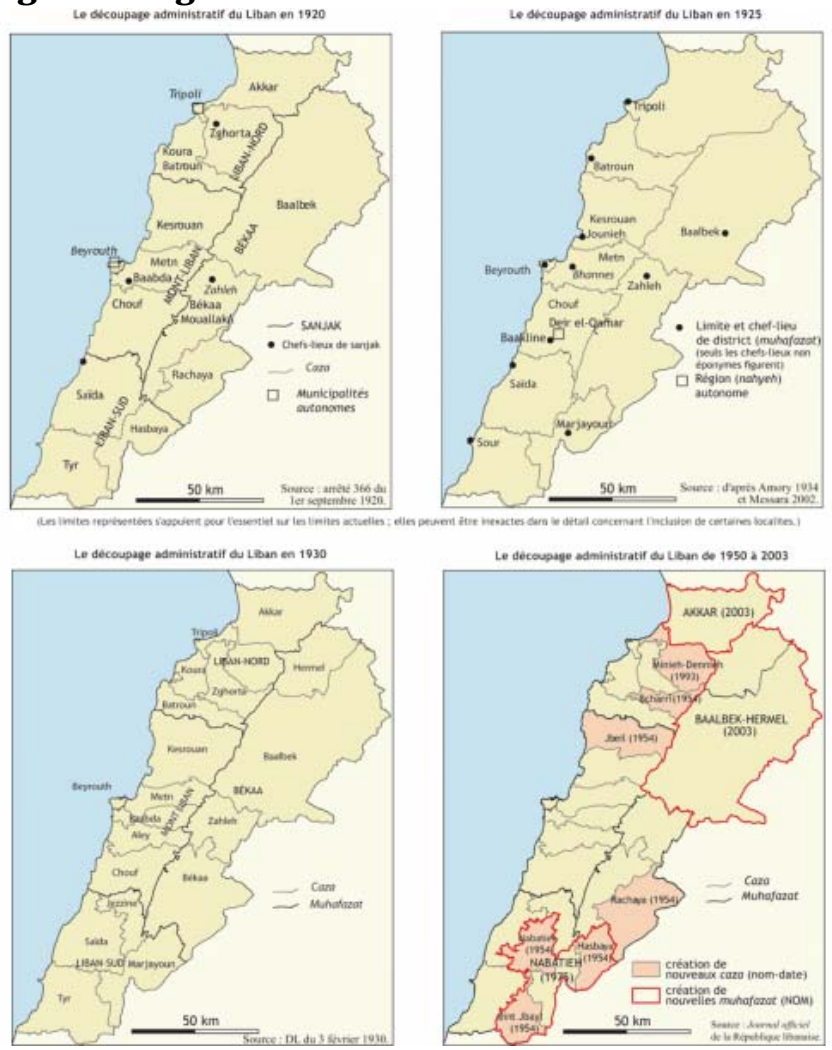

Vers le Grand-Beyrouth : urbanisme et politique des années 1946 à 1970

À partir des années 1930, mais surtout 1950, la croissance urbaine déborde nettement la municipalité de Beyrouth et la question se pose d'une planification urbaine extra-muros. Les plans de l'urbaniste suisse Egli, en poste à Beyrouth de 1946 à 1951, témoignent de ce dépassement, avec des aménagements routiers et des lotissements vers l'est, du côté de Sinn el-Fil et Dekouaneh, et vers le sud - notamment les Sables, qu'on appelle alors la 
banlieue sud-ouest. Ces plans se traduisent, sur le plan administratif et politique, par la montée en puissance de l'administration des municipalités au sein du ministère de l'Intérieur, qui concentrait jusqu'alors les prérogatives d'urbanisme. Les municipalités de banlieue, souvent de simples villages, contestent certains de ces projets, notamment en banlieue sud avec le désormais célèbre procès " des Sables », en 1953 (CleRcHUYBRECHTS 2008). À cette époque, c'est la dénomination de Beyrouth et ses banlieues (Bayrout wa dawahiha) qui prévaut.

L'avènement du chéhabisme se traduit par une centralisation plus forte des enjeux urbanistiques, l'aménagement du territoire constituant un outil privilégié par le régime pour assurer sa légitimité. En région, cela s'exerce par le biais des projets de l'IRFED (équipement en infrastructures et déploiement de l'administration); dans la capitale et ses banlieues, deux plans successifs apparaissent, liés l'un à l'autre par Écochard : le plan des cités gouvernementales et celui de Beyrouth et ses banlieues. C'est dans ce contexte qu'éclate une intéressante polémique sur le nom à donner à l'agglomération de Beyrouth. Dans les études menées par l'IRFED en 1959-1960 apparaît - pour la première fois à ma connaissance - la notion de Grand-Beyrouth, mais sous la forme d'un mixte francoanglais : « Great Beyrouth » (Mission IRFED - Liban 1959). En 1961, Écochard obtient du gouvernement un décret instituant un périmètre du Grand-Beyrouth (Bayrout al-Kobra) placé sous la supervision d'une commission chargée d'en effectuer l'étude d'urbanisme et de définir des propositions. Cette création institutionnelle suscitera deux débats bien distincts.

8 Le premier occupe la sphère technique. L'urbaniste Saba Shiber critique la délimitation retenue par Écochard et propose un Greater Beirut prenant en compte les régions dans l'orbite quotidienne de la capitale sur le plan des migrations alternantes. Est-ce en réponse à cette critique ? Le plan finalement livré en 1963 englobe toute la zone limitée par l'isoligne de $450 \mathrm{~m}$, entre le Nahr el-Kalb et le sud de Khaldeh.

Fig. 3 et 4 : deux délimitations concurrentes : Beyrouth et ses banlieues et Greater Beirut

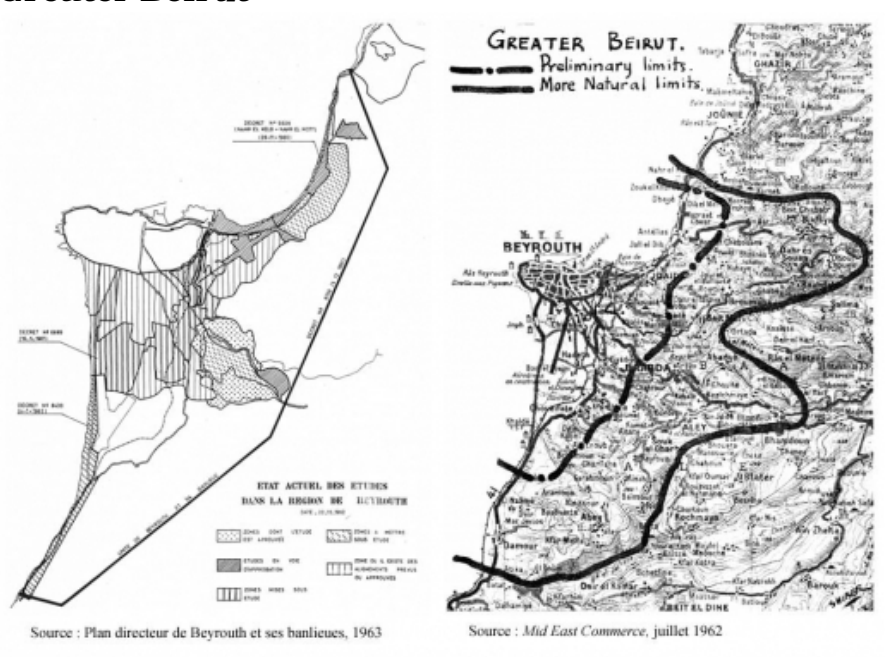

Le second débat est de nature politique. Quelques mois après la création de l'entité appelée «Grand-Beyrouth », celle-ci change de nom et devient "Beyrouth et ses banlieues » - c'est ainsi qu'est connu le plan d'Écochard. Si l'on suit la lecture de Saba Shiber, ce changement traduirait un double refus : celui des municipalités de banlieue, qui refusent de tomber sous la coupe de la capitale ou, à travers le Grand-Beyrouth, de l'État. Dans un contexte où Saeb Salam figure au premier rang des opposants politiques de Chehab, même s'il fut un temps son premier ministre ; mais celui de la municipalité de Beyrouth, qui refuse de voir son autonomie bridée par cette même tutelle.

\section{Du Grand-Beyrouth à la RMB : urbanisme et politique durant la guerre civile}

11 Un deuxième moment de conflit autour de l'émergence d'une entité administrative ou opérationnelle représentant l'agglomération de Beyrouth est celui de l'étude du Schéma directeur de la région métropolitaine de Beyrouth. Cette nouvelle dénomination renvoie à un nouveau concept territorial promu par une équipe franco-libanaise associant la Direction générale de l'urbanisme (DGU), le Conseil pour le développement et la 
reconstruction (CDR) et l'IAURIF.

L'origine de cette coopération remonte au début des années 1970, même si l'Institut d'aménagement et d'urbanisme de la région parisienne (IAURP) est déjà intervenu au Liban, brièvement, en 1965. Entre 1971 et 1973, il s'agit de préparer un schéma directeur pour Beyrouth aux horizons 1985 et 2000. L'enjeu est de remettre à jour les études d'Écochard en fonction des nouveaux développements et surtout, de renouveler les méthodes et les outils. À cet égard, la coopération entre les deux institutions et leur produit, le Livre blanc, aboutit à l'application de méthodes innovantes à Beyrouth, sur le plan statistique comme celui de l'analyse de l'occupation des sols. Elle traduit également l'émergence de nouvelles préoccupations, en particulier environnementales. Le document produit (République, Direction générale de l'urbanisme libanaise 1973) n'est pas un plan d'urbanisme mais une réflexion sur l'avenir, à travers divers scénarios étudiés en détail, et aboutit à des orientations à l'échelle de l'ensemble du pays.

13 Une nouvelle acception de la notion de Grand-Beyrouth y est proposée. Il s'agit du reste d'un territoire non seulement élargi vers les banlieues nord de J ounieh, mais aussi aux franges floues, en particulier dans la montagne, comme le traduit cette représentation graphique (fig. 5). En effet, aux yeux des urbanistes, le rayonnement de la capitale se mesure à l'échelle du pays - la Békaa centrale et une grande partie du littoral apparaissent comme déjà soumises à l'attraction beyrouthine. Dès lors, point n'est besoin de limites fixes ; celles-ci n'ont d'usage que pour les computations statistiques. Par ailleurs, le document n'aborde pas du tout la question institutionnelle : cette région floue n'est donc pas associée à un pouvoir ou une administration quelconque.

\section{Fig. 5 : Le Grand Beyrouth selon le Livre blanc}

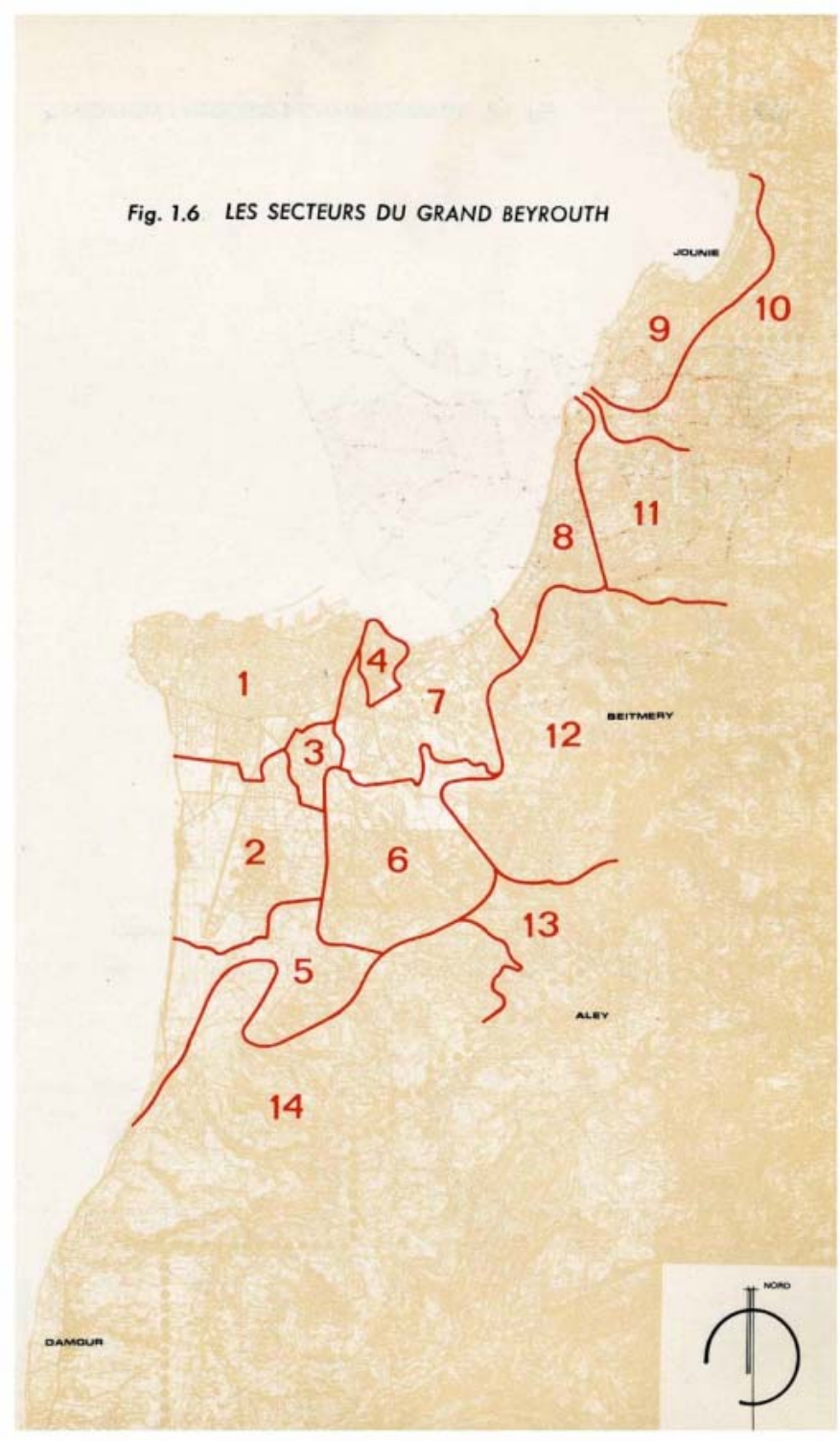


raison des déplacements forcés de population et des destructions de logements et d'infrastructures. À deux reprises, en 1977-1978 et 1982-1983, des pauses durables dans le conflit conduisent à l'étude de nouveaux projets d'urbanisme, pour reconstruire les parties de la ville les plus endommagées et pour planifier le développement futur. Seulement esquissée en 1977, l'élaboration d'un schéma directeur est relancée en 1983, au début de la présidence d'Amine Gemayel. L'IAURIF est de nouveau sollicité et, aux yeux de tous les participants, il s'agit autant d'une intervention nécessitée par la situation dramatique du Liban et de sa capitale que d'une poursuite du projet entamée quelques années auparavant. Plusieurs des acteurs de cette équipe sont d'ailleurs les mêmes, notamment le chef d'équipe français, Jacques Piétri. Si le directeur de la DGU de l'époque, Mohamed Fawaz, n'avait pas été impliqué dans le Livre blanc, il avait été régulièrement en contact avec l'IAURP par la suite. D'autres participants incarnent la continuité, comme le statisticien Abdou el-Kahi.

Le Schéma directeur de la région métropolitaine de Beyrouth (SDRMB) a fait couler beaucoup d'encre dans les publications des années de la reconstruction (fig. 6) : nombre d'urbanistes et d'architectes au Liban l'ont considéré comme le produit d'un compromis, voire même d'une compromission des urbanistes et de l'administration dans un contexte de guerre civile et de toute-puissance des milices. La mise en avant du concept de région métropolitaine de Beyrouth, pour remplacer le concept de Grand-Beyrouth, a été lue comme le paravent technocratique de ce renoncement (TABET 1991 ; BEYHUM 1997 ; SARKIS 1998). Mais dans le même temps, la notion de Grand-Beyrouth connaissait pour la première fois un usage politique (DAVIE 1993 ; KIWAN 1991). En effet, la sécurisation du Grand-Beyrouth, c'est-à-dire le désarmement ou le retrait des milices et leur remplacement par diverses forces militaires (armée libanaise mais aussi force arabe de dissuasion en 1977 et surtout force multinationale en 1982), est devenue un enjeu central de négociation pour un cessez-le-feu et la pacification du pays.

\section{Fig. 6 : Schéma directeur de la région métropolitaine de Beyrouth (SDRMB)}

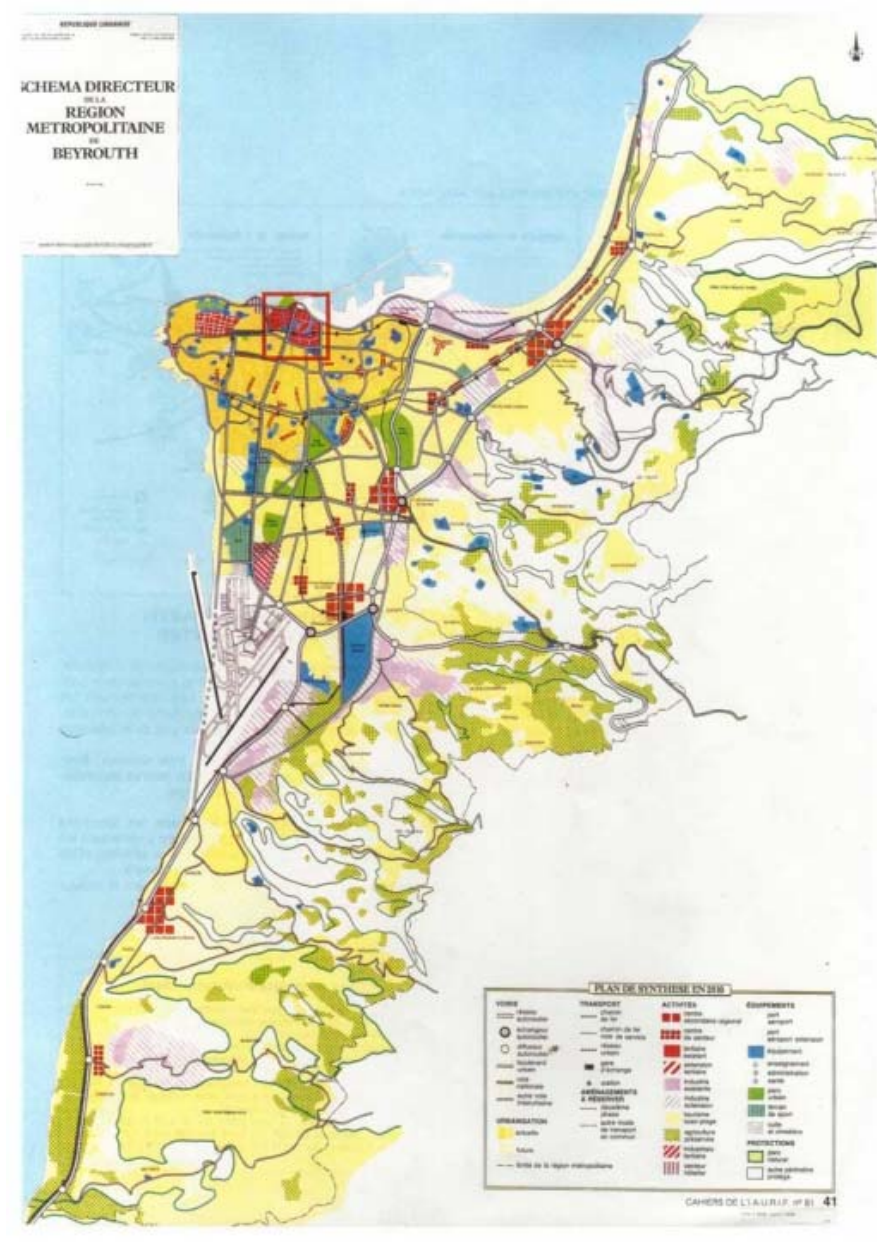

16 Pour l'architecte Hashim Sarkis, la délimitation de la Région métropolitaine de Beyrouth (RMB) était très contestable, notamment par son exclusion de la zone de Jounieh et d’autres localités de montagne. Selon lui, elle était due à la volonté de parvenir à un 
équilibre démographique entre chrétiens et musulmans au sein de l’agglomération. Or ces localités étaient de peuplement chrétien, et leur inclusion aurait contribué à un surnombre chrétien dans l'agglomération. Il dénonçait chez les urbanistes une obsession typiquement orientaliste consistant la société au seul filtre de l'appartenance confessionnelle (SARKIS 1998).

L'argument n'est qu'à moitié recevable : en réalité, la RMB comptait déjà en 1983, une majorité de chrétiens, entre 60 et $65 \%$ selon environ (VERDEIL 2002) ; en réalité, l'inclusion de ces localités n'aurait donc que fortement accentué le déséquilibre démographico-confessionnel déjà existant.

Mon enquête auprès des protagonistes de ce schéma d'urbanisme m'a surtout conduit à nuancer cette vue (VERDEIL 2002). D'une part, la limite sud de la RMB constitue une extension du périmètre du Livre blanc. Il s'agissait d'inclure Damour, village chrétien rasé en 1976 par les forces palestiniennes (en représailles aux massacres perpétrés dans les camps), inclusion qui, pour le président phalangiste Gemayel, s'imposait. En revanche, l'exclusion du Kesrouan au nord semble plutôt due, aux dires de l'architecte et urbaniste Pierre el-Khoury, alors ministre des Travaux publics et l'un des grands promoteurs de ce projet au sein du gouvernement, au refus des Forces libanaises de s'inscrire dans une initiative pilotée par le président Gemayel, dont ce parti était alors un adversaire politique. Quant aux limites en altitude de cette RMB, elles s'inscrivaient dans la continuité des exercices de planification antérieurs. On peut sans doute aussi les lire comme une volonté de restreindre la zone de planification au secteur directement contrôlé par le président Gemayel, secteur dans lequel contrôle politique et urbanisme constituaient en quelque sorte les deux faces d'une même volonté d'hégémonie. C'est ce qui fut exprimé par l'Intifada du 6 février 1984, qui se déclencha en réaction à une intervention urbanistique directement menée par l'armée libanaise, fidèle à Gemayel, consistant à démolir divers baraquements et constructions illégaux dans la banlieue sud. Loin de n'être que fantaisie orientaliste, la définition de ces limites et le glissement de dénomination renvoient donc à des enjeux politiques structurants, bien que masqués.

\section{Conclusions intermédiaires}

19 À travers ces deux épisodes, deux conclusions peuvent se dégager. Les urbanistes des administrations étatiques et leurs collègues consultants, locaux ou étrangers (notamment français) ont largement communié dans l'idée de la nécessité d'un cadre institutionnel adapté et en tout cas, à défaut de ce qui n’a jamais réellement été tenté, d'une vision urbaine à l'échelle de l'agglomération. Cette vision surplombante a été associée non pas avec un pouvoir supramunicipal, mais avec celui d'une administration étatique centralisée, historiquement constituée pour asseoir, par l'urbanisme, le pouvoir de l'État contre certains groupes sociaux et notamment les « spéculateurs locaux » et les municipalités ; ces dernières étaient pensées comme responsables des dérives de l'urbanisation, dont elles auraient par exemple toléré les débordements populaires et illégaux (c'est très clair à propos de la banlieue sud) ${ }^{1}$. Avec la guerre civile, cette figure du pouvoir local anarchique - ou plutôt vecteur d'anarchie - s'est complexifiée avec l'ajout des pouvoirs miliciens, protecteurs des empiétements illégaux. Le corollaire de cette vision surplombante, surtout dans un contexte de guerre, mais c'est vrai également des années 1950-1970, est une extrême difficulté à faire émerger et à accepter de reconnaître les discours et les aspirations des citoyens ou des municipalités dans l'analyse des besoins et dans les projets prioritaires. Par-delà les différences de contexte, le point commun entre ces interventions - les appellations elles-mêmes en rendent compte - réside dans leur vision technocratique de l'avenir et dans des solutions d'aménagement ne laissant place à aucune dimension démocratique.

\section{Appréhender le Grand-Beyrouth, entre morphologie et fonctionnalité, tentatives récentes}

20 Étudions à présent une série de tentatives de délimitation de l'agglomération de Beyrouth, émanant soit de géographes, soit d'urbanistes qui se situent moins directement dans la dimension de projet d'aménagement, tout en essayant d'en comprendre également la dimension normative. Revenons sur les tentatives de délimitation du Grand- 
Beyrouth relevant a priori davantage de la modélisation et de l'analyse que de la planification. Il s'agira alors de voir comment ces exercices s'inscrivent dans la tension savoir-dénomination-politique identifiée ci-dessous.

21 Mentionnons d'abord deux tentatives de délimitation des banlieues de Beyrouth reposant sur des méthodologies novatrices par deux géographes. La première est celle, bien connue, des « banlieues-accordéon » d'André Bourgey (BOURGEY 1970). Pour identifier l'enveloppe beyrouthine, il fonda son analyse sur les mobilités quotidiennes mais aussi saisonnières de la population. En prenant en compte l'estivage, il donnait des délimitations de la banlieue de Beyrouth une vision dynamique dans le temps. De son côté, Liliane Barakat basa son enquête sur les perceptions d'enfants des écoles libanaises (BARAKAT 1987).

22 Toutefois, la grande majorité des travaux postérieurs s'inscrivit dans une approche morphologique, permise par l'émergence des techniques d'imagerie satellitaire ou aérienne, avec une visée expérimentale et méthodologique (IAURIF 1989 ; DAVIE et DROUOT 1996 ; ARNAUD 1997 ; HUYBRECHTS 1997 ; HUYBRECHTS 2001 ; ZAAROUR et al. 2001). Ces tentatives pionnières des années 1990 ont ensuite cédé le pas à des approches plus systématiques et robustes, couplant délimitation spatiale et dénombrement démographique.

23 La première est celle mise en œuvre par l'équipe responsable de l'élaboration du Schéma directeur d'aménagement du territoire libanais (SDATL) (DAR-IAURIF 2003). Sur la base d'images satellites datant de 1998, une identification des espaces urbanisés a été menée. Couplé aux estimations démographiques à l'échelle locale, ce travail a permis de sortir de la conception dominante de la RMB qui avait prévalu dans l'administration durant toutes les années 1990. Cette nouvelle réalité urbaine fut nommée par le SDATL « l'aire urbaine centrale » et sa population estimée à 1,65 million d'habitants (fig. 7). Cet ensemble était cependant composé d'entités distinctes et les polarités urbaines classiques de J ounieh, Aley, Broumana, Beit Mery, Bikfaya, etc. étaient mises en évidence, certains de ces noyaux étant appelés à jouer un rôle dans la structuration urbaine future de l'agglomération. Les travaux du SDATL eurent également pour effet de révéler l'existence de polarités urbaines nouvelles et jusqu'alors invisibles, telles que le curieux ensemble constitué par la conurbation des villages de Barja, Chhim et leurs environs, au sud de l'agglomération, évaluée à une population d'environ 60000 habitants, et que bien peu de Libanais identifient comme une ville. Du reste, la forme de cette nouvelle agglomération est de type rhizomatique et défie les classifications habituelles. Cet ensemble apparaît comme une banlieue-relais inédite de Beyrouth, notamment grâce à l'autoroute du sud qui permet des connexions améliorées vers la métropole.

Fig. 7 : l'aire urbaine centrale dans le Schéma directeur d'aménagement du territoire libanais (SDATL) 


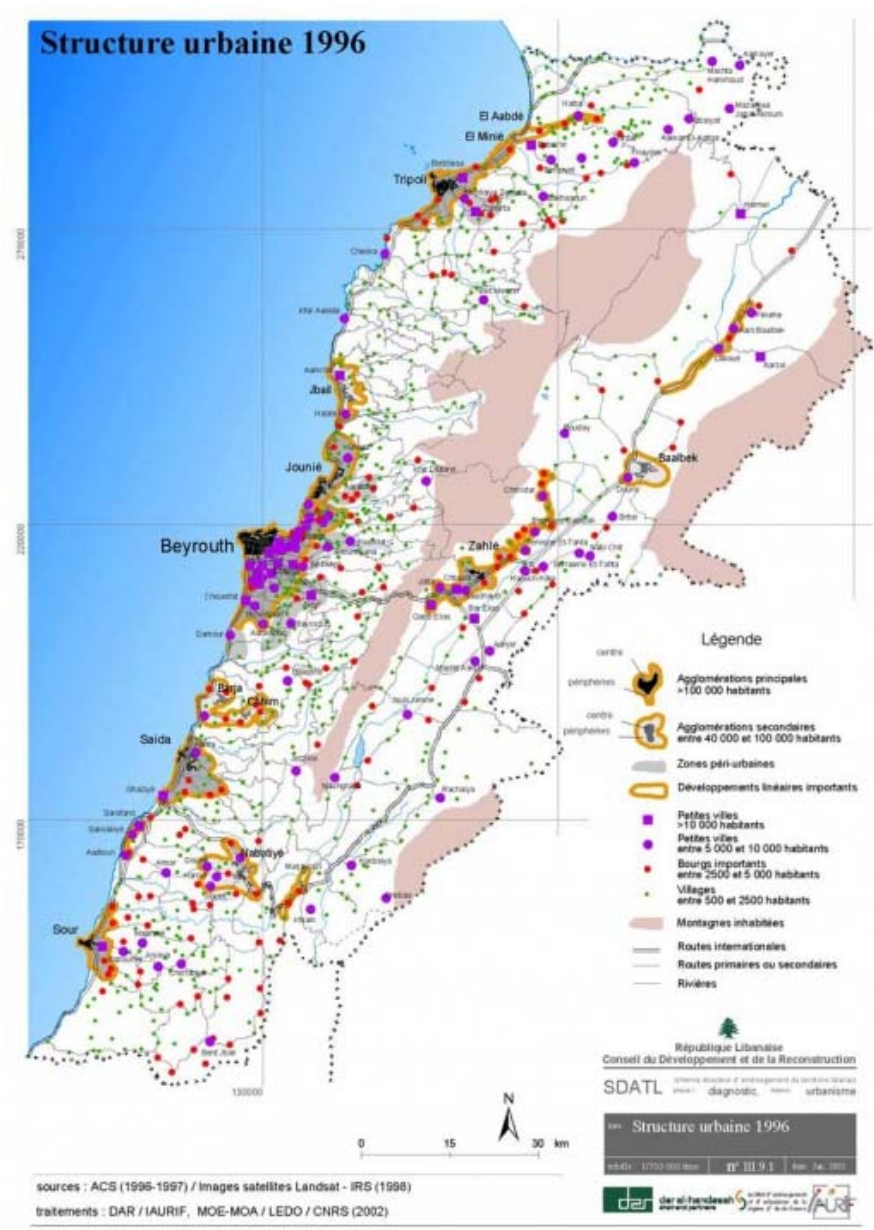

24 On peut faire des remarques similaires à propos des délimitations des zones urbaines effectuées par le groupe de recherche Géopolis, récemment publiée (GAZEL, HARRE, et MORICONI-EBRARD 2011). Là encore, l'agglomération apparaît comme une pieuvre ou un ensemble racinaire partant à l'assaut des crêtes surplombant Beyrouth. Toutefois, les premiers travaux effectués selon cette méthodologie, due à J ean-Luc Arnaud, avaient déjà montré, sur les cartes de l'armée libanaise de 1963, ce type d'extensions (ARNAUD 1997). L'une des originalités de la base Géopolis est le principe d'absorption des entités urbaines préexistantes dans le continuum urbain. Ces localités, telles Aley, Jounieh, J beil, progressivement incluses dans l'agglomération capitale, perdent alors leur nom et leur individualité. En même temps, aucune réalité fonctionnelle n'est prise en compte ici, par exemple les migrations alternantes, alors que dans la logique du SDATL, elles constituaient un critère (parmi d'autres) d'individualisation de pôles urbains internes à l'agglomération. Par ailleurs, le nom de l'agglomération est devenu simplement Beyrouth et non plus Grand-Beyrouth.

Fig. 8 : L'agglomération de Beyrouth selon les travaux de Géopolis (e-geopolis.eu) 


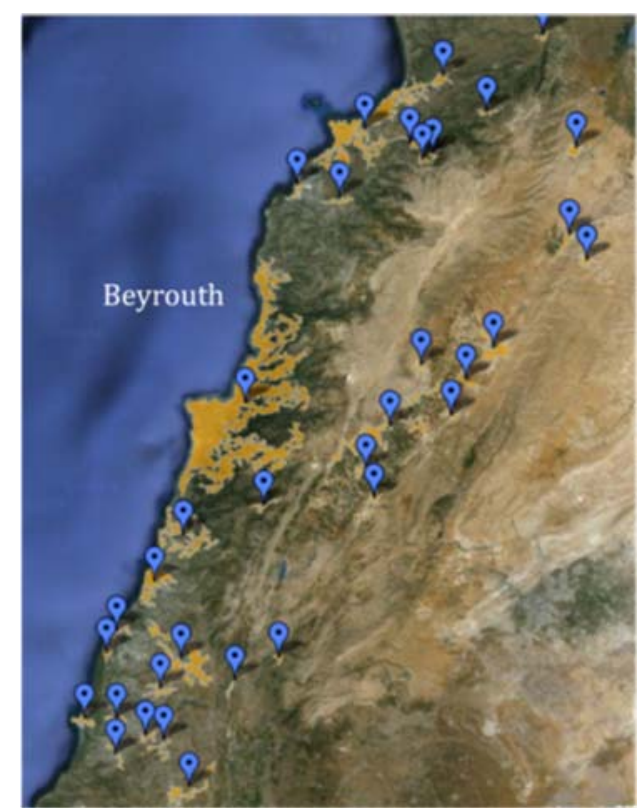

La particularité de ces deux exemples, lorsqu'on les met en comparaison avec les épisodes antérieurs, c'est que ces exercices de délimitation sont largement déconnectés de projets, en particulier pour Géopolis. Certes, le SDATL fait des propositions d'aménagement, mais ce sont des principes à une échelle plus large et la délimitation n'est pas celle d'un territoire et d'une logique de projet. Ce qui relie les deux approches est en fait la dimension prospective, puisque ces délimitations sont associées à des projections de croissance urbaine pour le futur. La délimitation n'est donc plus renvoyée à une finalité directement pratique et appliquée, et constitue une réalité dynamique et instable.

\section{Émergence de nouvelles logiques de découpage des territoires urbains}

\section{Les nouveaux enjeux de la gouvernance des banlieues}

26 Aujourd'hui la volonté d'appréhender globalement Beyrouth et ses banlieues, et de traiter cette entité comme un territoire de projet et d'aménagement, ne semble plus à l'ordre du jour pour les administrations (le CDR, la DGU ou même la CAS). Cette dernière partie examine des périmètres renvoyant à d'autres instances de gestion urbaine, jusqu'alors peu prises en considération dans les réflexions urbanistiques : les municipalités et les opérateurs de services urbains.

27 La question urbaine est transformée au Liban par la réémergence des municipalités depuis les élections de 1998. Certes, cette réémergence est problématique et faute d'aller de pair avec une allocation de ressources correspondantes, les constats du début de période (FAVIER 2001) restent largement valables. Néanmoins, les municipalités jouent un rôle croissant dans les questions d'aménagement comme un certain nombre de travaux commencent à le reconnaître (FARAH 2011 ; YASSIN 2011). Je voudrais ici particulièrement m'intéresser à la mise en place de nouveaux territoires d'action, les unions de municipalités, par lesquels les municipalités libanaises tentent de regrouper leurs moyens pour réaliser des projets communs complexes, notamment dans le domaine des services urbains (déchets) ou celui de la planification. Ce mouvement a connu une forte accélération depuis le début des années 2000 et la relance des municipalités depuis 1998. Le nombre d'unions est passé de 20 en 2003 à 42 en 2010 (YASSIN 2011; VERDEIL, FAOUR et VELUT 2007).

\section{Fig. 9 : Les unions de municipalités au Liban en 2010}




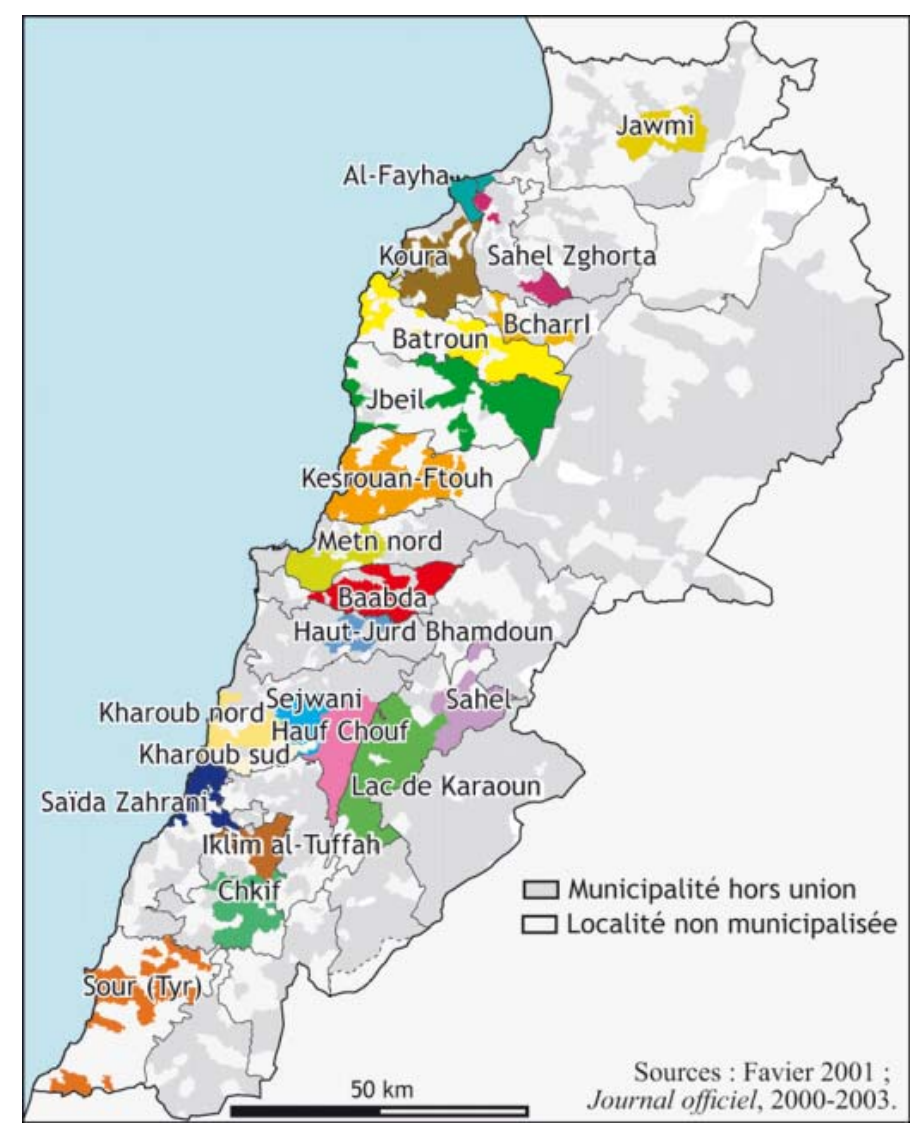

Verdeil, Faour et Velut, Atlas du Liban : territoires et société, Ifpo, 2007

L'examen de la carte des unions de municipalités (fig. 9) permet d'observer un mouvement de création institutionnelle qui a affecté notamment les banlieues de Beyrouth du caza de Baabda. D’autres banlieues avaient déjà des formes de coopérations plus anciennes, comme le Metn nord ou le Kesrouan (années 1980). Légalement, les coopérations municipales doivent forcément s'inscrire dans les limites de cazas, ce qui rend de toute façon impossible l'émergence d'un ensemble « banlieues de Beyrouth ». D'autre part, même si leur objet est technique, il apparaît clairement que ces unions renvoient à des formes de coopération où le politique tient une grande part.

Historiquement par exemple l'émergence de l'union des municipalités du Metn nord est due aux initiatives d'Amine Gemayel, député de cette région, durant les années de guerre civile précédant son mandat. Plus récemment, l'union a été dominée par la famille Murr (VERDEIL 2002).

30 Dans le caza de Baabda, l'émergence des nouveaux périmètres d'action supramunicipale s'analyse également à la lumière des enjeux politiques au sein de la banlieue sud et sud-est (FARAH 2011). Farah a bien montré leur complexité, sans les réduire à la dimension communautaire, mais en soulignant leur lien avec la nature des élites politiques (familles vs parti). Lancées dans le début des années 2000, les tentatives de construire une union pour toute la banlieue ont échoué sur la mobilisation partisane et communautaire postérieure à la guerre de 2006, qui a vu la création d'une union correspondant aux quatre municipalités de la banlieue sud dominée par le Hezbollah, tandis que les banlieues est suivaient quelque temps après (fig. 10). Mais cette mobilisation correspondait également à une convergence d'intérêt de ces municipalités dans leur relation avec l'État libanais sur la partie ouest de leur territoire, concerné par le projet Elyssar, dans lequel elles cherchent à affirmer leur présence.

Fig. 10 : Les unions municipales dans le Sahel (banlieue sud et sud-est de Beyrouth) 


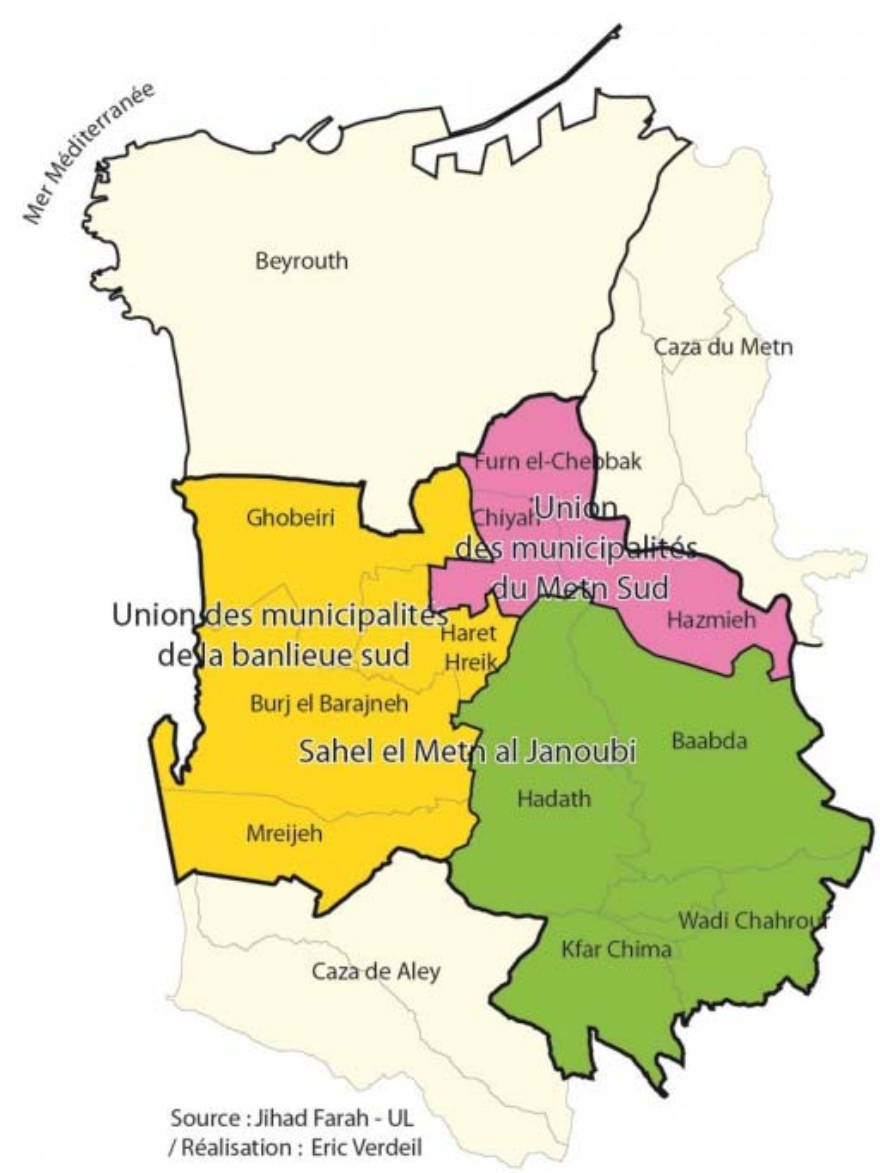

31 Ces constructions territoriales ne sont pas qu'affaire de délimitation administrative et politique : les noms de ces nouvelles entités sont également lourds de signification et renvoient à des légitimités sociales en concurrence. Le nom historique de la zone est en effet le Sahel du Metn sud (Sahel al-Metn al-J anoubi), c'est-à-dire le littoral de la région du Metn sud, désignation géographique ancienne du caza aujourd’hui désigné par le nom de son chef-lieu, Baabda. L'appellation de Sahel al-Metn al-J anoubi ne comporte aucune référence à Beyrouth et révèle la continuité dans l'indépendance administrative des élites politiques locales par rapport à la grande ville voisine. C'est ce nom qui avait été retenu initialement pour nommer l'union municipale censée regrouper toutes les municipalités de la banlieue, et qui est resté pour l'union des municipalités de la partie est du secteur - ces municipalités étaient toutes à majorité chrétienne et souvent dirigées par les élites locales issues des familles traditionnelles.

À l'inverse, pour l'union des quatre municipalités du secteur ouest, on retient le nom Unions des municipalités de la banlieue sud (al-Dahiya al-janoubiya). Ce nom s'oppose à la fois à la dénomination du secteur dans les anciens projets d'extension de l'État, qui évoquait les banlieues au pluriel, et à l'appellation du Sahel. Mona Harb a montré comment ce toponyme de Dahiya s'est progressivement imposé dans l'histoire récente de la ville pour désigner un territoire à majorité chiite, avec une forte dimension péjorative et stigmatisante à l'encontre d'une banlieue vue comme illégitime dans son développement urbain et perçue comme une menace politique (HARB 2003). De ce point de vue, l'adoption de ce toponyme semble représenter un retournement du stigmate et l'affirmation fière d'une communauté d'intérêts comme celle d'une identité pleinement assumée.

\section{Les périmètres de gestion des services urbains}

33 Mentionnons en dernier lieu d'autres types de périmètres opérationnels qui structurent l'action urbaine, et en particulier les périmètres liés à la gestion des services urbains. Ces enjeux sont souvent absents de la réflexion sur l'avenir de l'agglomération, qui privilégie les questions d'espace bâti et la planification classique. De fait, les services urbains sont en général invisibles - c'est le cas lorsque le service est bien rendu : les poubelles sont ramassées et ne restent pas dans la rue, l'électricité ou l'eau passent en souterrain ou par 
des fils discrets. En fait, la visibilité des services urbains surgit lorsqu'ils ne fonctionnent pas : rues encombrées d'écheveaux de fils électriques, réservoirs, pompes et camionsciternes donnant à voir un circuit parallèle de la distribution d'eau dans la ville, etc. Pour les citoyens, périmètres et modes de gestion de ces services urbains restent eux-mêmes opaques, déterminés par des techniques et des accords financiers qui ne font généralement pas l'objet de débats politiques. Pourtant, là également, l'action collective s'inscrit dans des périmètres aux effets structurants (KAIKA et SwYNGEDOUW 2000 ; J AGLIN 2004).

On peut illustrer ce point à travers la question de la distribution électrique, dont l'inégalité de distribution au Liban est particulièrement frappante. La capitale intramuros, Beyrouth, est particulièrement privilégiée avec des coupures qui ne durent actuellement que 3 heures par jour. De 2000 à 2006, elle bénéficiait de l'électricité 24 heures sur 24. À l'inverse, les autres régions du pays et notamment les banlieues sont bien mal servies (VERDEIL 2009). Ces banlieues sont regroupées en deux zones, Chiyah et Antélias, correspondant aux banlieues sud et est. Historiquement, Beyrouth et ses banlieues correspondaient à une seule région opérationnelle, mais cette unité (au traitement interne fortement différencié) est maintenant remise en cause avec les transformations de gestion mises en œuvre par le ministère de l'Énergie. La distribution (gestion du réseau de distribution, facturation et collecte, lutte contre la fraude) est en effet confiée à des entreprises privées mises en concurrence. Afin de délimiter des zones aux profils de consommation équilibrés, les regroupements opérés associent diverses régions sans considération d'unité géographique, sociale ou confessionnelle au contraire. Ainsi, le territoire municipal de Beyrouth sera associé à la Békaa, la zone dite de Chiyah (banlieues sud et sud-est) sera associée aux cazas du Sud du Mont Liban et aux deux régions méridionales du Liban, tandis que les banlieues est et nord de Beyrouth sont regroupées avec les cazas du Nord du Mont Liban et le Liban nord (fig. 11).

Fig. 11 : Les nouveaux périmètres de la gestion de la distribution électrique au Liban

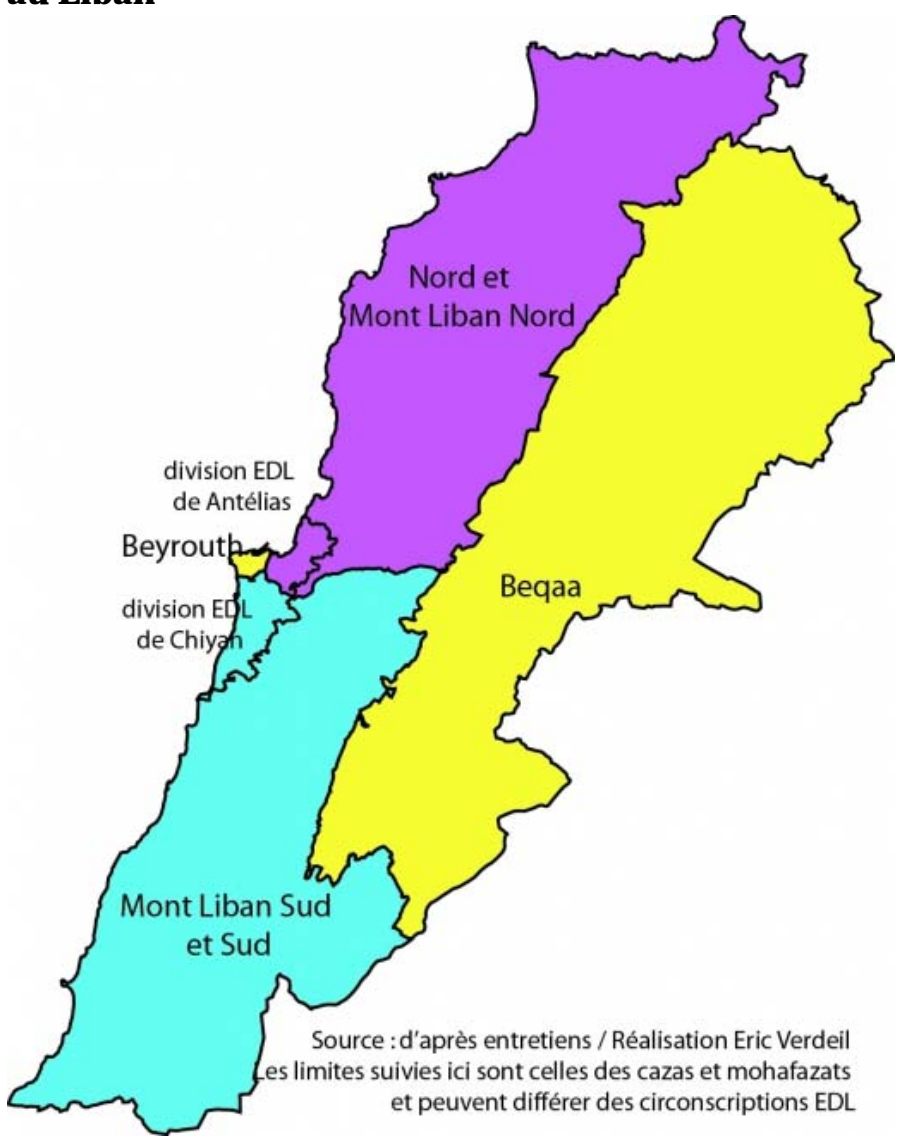

35 Les deux exemples analysés ici soulignent la diversité des périmètres gestionnaires et montrent que leur construction obéit à des logiques très diverses, où considérations économiques et techniques se mêlent aux enjeux politiques. Il apparaît qu'au Liban, la référence à une métropole organique et polarisée n'est plus guère mobilisée dans l'action publique, qui se fonde sur d'autres ressorts. La diversification des acteurs participant à 
l'action publique constitue notamment un fait majeur, aujourd'hui devenu légitime et qui contribue justement à rendre obsolète cette référence à la métropole unifiée.

\section{Conclusion}

36 J'ai concentré mon attention sur différents découpages de la métropole beyrouthine et sur leurs dénominations, en cherchant à comprendre ce que cela révélait de la perception des banlieues de Beyrouth par les urbanistes, les experts de l'urbain ainsi que les acteurs politiques impliqués. Ces constructions territoriales sont pour une large part restées confinées au sein même des sphères savantes ou technocratiques et n'ont guère fait l'objet d'appropriations par la population libanaise. Elles témoignent de la longue durée d'une représentation qui voit l'État comme seule instance légitime de gestion et de mise en ordre de l'organisation urbaine, ce discours reflétant la prééminence étatique et ses avatars technocratiques dans l'énonciation du futur urbain. À cet égard, l'histoire des découpages et des dénominations s'inscrit largement dans une tendance plus large, celle de la quête du périmètre idéal qui a dominé l'histoire de l'urbanisme européen (VANIER 2008). En bien ou en mal, cette tentation ne paraît plus d'actualité. Elle conduit les chercheurs à déplacer la focale vers d'autres enjeux et logiques de fabrication de l'action urbaine, qu'illustrent aujourd'hui les transformations et les recompositions de la politique municipale ou de la gestion des services urbains.

\section{Bibliographie}

ARNAUD, Jean-Luc, 1997, « Trop grand Beyrouth ? », dans Beyrouth, Grand-Beyrouth, J ean-Luc (dir.), Les cahiers du CERMOC 16, Beyrouth, CERMOC, pp. 209-227.

BARAKAT, Liliane, 1987, « La perception de Beyrouth et de sa périphérie urbaine (B.P.U.) par des écoliers libanais », Annales de géographie de l'Université Saint-J oseph 8, Beyrouth, pp. 101-135.

BEYHUM, Nabil. 1997, « Petit manuel de la reconstruction à Beyrouth », dans Beyrouth : regards croisés, Michael F. Davie (dir.), Villes du monde arabe 2, Tours, URBAMA, pp. 253-272

BOURGEY, André, 1970, « Problèmes de géographie urbaine au Liban », Hannon, revue libanaise de géographie 5, pp. 97-123.

Central administration of statistics (CAS), 2008, Living Conditions Survey 2007, Beirut, Central Administration of Statistics.

CleRc-HuYBRECHTS, Valérie, 2008, Les quartiers irréguliers de Beyrouth : Une histoire des enjeux fonciers et urbanistiques dans la banlieue sud, Presses de l'Ifpo, [En ligne] http://ifpo.revues.org/ 63.

DAGoRN, René, 2003, " Métropole/ Mégalopole », dans Dictionnaire de la géographie, J acques Lévy et Michel Lussault (dir.), Paris, Belin, pp. 609-612.

DAR-IAURIF, 2003, Schéma Directeur d'Aménagement du Territoire Libanais (SDATL), Phase 1. Diagnostics et Propositions. Rapport définitif, Beyrouth, Conseil du développement et de la reconstruction, janvier.

DAVIE, Michael F., et J ean-Louis DROUOT, 1996, « La périphérie urbaine et les extensions de la ville de Beyrouth (Liban) : étude par traitement d'une image SPOT », Cybergeo : European Journal of Geography 157, Dossiers, [En ligne] http:// cybergeo.revues.org/ 719.

DAVIE, Michael F.,1993, « Quelles limites géographiques pour Beyrouth ? », dans Beyrouth : construire l'avenir, reconstruire le passé ?, Nabil Beyhum, Assem Salam, et Jad Tabet (dir.), Dossiers de l'Urban Research Institute édités par le support de la Ford Foundation, Beyrouth, Urban Research Institute. 
DEPAULE, J ean-Charles (dir.), 2006, Les mots de la stigmatisation urbaine », Les mots de la ville 4, UNESCO/Maison des sciences de l'homme.

FARAH, Jihad, 2011, Différenciations sociospatiales et gouvernance municipale dans les banlieues de Beyrouth : à travers l'exemple de Sahel AlMatn AlJanoubi et des municipalités de Chiyah, Ghobeiri et Furn AlChebbak, Université de Liège, [En ligne] http:// orbi.ulg.ac.be/ handle/ 2268/94024.

FAVIER, Agnès (dir.), 2001, « Municipalités et pouvoirs locaux au Liban », Les cahiers du CERMOC 24, Beyrouth, CERMOC.

FAWAZ, Mona, 2008, " An Unusual Clique of City-Makers: Social Networks in the Production of a Neighborhood in Beirut (1950-75) », dans International J ournal of Urban and Régional Research 32 (3) (septembre), pp. 565-585.

GAZEL, Hervé, Dominique HARRE, et François MORICONI-EBRARD, mai 2011, « Tableau de bord Liban », L'urbanisation des pays du Moyen-Orient et de l'Afrique du Nord (MENA), 1950-2030 26, dans e-géopolis, [En ligne] http://e-geopolis.eu/menapolis /TABLEAU_BORD_LIBAN.pdf.

HARB, Mona, 2003, « La Dâhiye de Beyrouth : parcours d'une stigmatisation urbaine, consolidation d'un territoire politique », Gen 51(2), pp. 70-91.

HUYBRECHTS, Eric, 1997, « Densités beyrouthines ». Lettre d'information de l'ORBR 7, pp. 14-17.

HUYBRECHTS, Eric, 2001, « Les extensions urbaines de la région métropolitaine de Beyrouth ». Lettre d'information del'ORBR 13, pp. 10-14.

IAURIF, 1989, Cadrage régional de Beyrouth, CDR/ DGU/IAURIF.

J AGLIN, Sylvy (dir.), 2004, « Services en réseaux, services sans réseaux dans les villes du Sud ». Flux n 56-57, p. 140.

KAIKA, Maria, et Erik SwYNGEDOUW, 2000, « Fetishizing the modern city: the phantasmagoria of urban technological networks », International J ournal of Urban and Régional Research 24 (1) (mars 1), pp. 120-138.

KIWAN, Fadia, 1991, « Le Grand-Beyrouth, quel espace politique ? », dans Reconstruire Beyrouth. Les paris sur le possible, Nabil Beyhum (dir.), Études sur le monde arabe 5, Lyon, Maison del'Orient.

LAMARRE, Christine, et Catherine MAUMI, 2010, « Métropole », Dans L'aventure des mots de la ville, Christian Topalov, Laurent Coudroy de Lille, Jean-Charles Depaule, et Brigitte MARIN (dir.), Paris, Robert Laffont, pp. 749-753

MEURIOT, Paul, 1919, « Du concept de ville d'autrefois et aujourd'hui », La Vie urbaine (1 et 2), pp. 145-154.

Mission IRFED-Liban, 1959, Étude préliminaire sur les besoins et les possibilités de développement au Liban, Beyrouth, Mission IRFED-Liban.

MORICONI-EBRARD, François, 1993, L'urbanisation du monde depuis 1950, Paris, Economica-Anthropos, coll. Villes.

MORICONI-EBRARD, François, et J ean-Paul HuBERT, 2000, De Babylone à Tokyo : les grandes agglomérations du Monde, Gap, Ophrys, coll. Géophrys.

REEDER, David A, 2010, " Metropolis », Dans L'aventure des mots de la ville, Paris, Robert Laffont, pp. 768-774.

République, Direction générale de l'urbanisme libanaise, juin 1973, Livre blanc, Beyrouth 1985-2000, 2e éd., Beyrouth, DGU. 
RIVIĖRE D'ARC, Hélène (dir.), 2001 Nommer les nouveaux territoires urbains, UNESCO/Maison des sciences de l'homme, coll. Les mots de la ville.

SARKIS, Hashim, 1998, « Dances with Margaret Mead: Planning Beirut since 1958 », dans Projecting Beirut: Episodes in the Construction and Reconstruction of a Modern City 1, New York, Munich, Prestel, pp. 187-201

TABET, J ad, 1991, « La ville imparfaite », dans Reconstruire Beyrouth. Les paris sur le possible, Nabil Beyhum (dir.), pp. 85-120, Lyon, Maison de l'Orient, pp. 85-120

VANIER, Martin, 2008, Le pouvoir des territoires : essai sur l'interterritorialité, Paris, Géographie.

VERDEIL, Éric, 2002, Une ville et ses urbanistes : Beyrouth en reconstruction, Thèse de doctorat, Université Panthéon-Sorbonne (Paris).

VERDEIL, Éric, 2009, « Électricité et territoires : un regard sur la crise libanaise ». Revue Tiers Monde 198, p. 421-438.

VERDEIL, Éric, 2010, Beyrouth et ses urbanistes. Une ville en plans (1946-1975), Presses de l'Ifpo Beyrouth, [En ligne] http:// www.ifporient.org/ node/ 839.

VeRDEIL, Eric, Ghaleb FAOUR, et Sébastien VeluT, 2007, Atlas du Liban : territoires et société, Beyrouth, Presse de l'Ifpo.

YASSIN, Nasser, octobre 2011, Lebanon Urban Profile, Beirut, UN-Habitat.

ZAAROUR, Rita, Liliane BARAKAT, Janine SOMMA, et Nada SALIBA, 2001, « Extraction et suivi de la tache urbaine de l'agglomération de Beyrouth à travers une analyse satellitaire 1994-1998 : méthodes et limites », Géosphères, annales de géographie 21-22, p. 41-61.

\section{Notes}

1. Ceci bien que Mona Fawaz ait démontré comment les fonctionnaires de la DGU ont été eux-mêmes directement impliqués dans la production de cette urbanisation illégale (Fawaz 2008).

\section{Auteur}

Éric Verdeil

Chercheur, UMR Environnement Ville Société, CNRS, Lyon

Référence électronique du chapitre

VERDEIL, Éric. Délimiter les banlieues de Beyrouth. Des noms et des périmètres In : Des banlieues à la ville : Espaces et acteurs de la négociation urbaine [en ligne]. Beyrouth : Presses de l'Ifpo, 2013 (consulté le 03 septembre 2013). Disponible sur Internet : <http://books.openedition.org/ifpo/3457>. ISBN 9782351593394.

\section{Référence électronique du livre}

LONGUENESSE, Élisabeth (dir.) ; PIERI, Caecilia (dir.). Des banlieues à la ville : Espaces et acteurs de la négociation urbaine. Nouvelle édition [en ligne]. Beyrouth : Presses de l'Ifpo, 2013 (consulté le 03 septembre 2013). Disponible sur Internet : <http://books.openedition.org/ifpo/2826>. ISBN 9782351593394. 